\title{
REALIDAD DE LAS COMUNIDADES RURALES DE COSTA RICA
}

\author{
Erika Bonilla Houdelatth ${ }^{1}$ \\ Estudiante egresada de la Maestría en Educación Rural \\ Heredia, Costa Rica
}

Recibido 15 de junio 2007 • Aprobado 20 de agosto 2007

\begin{abstract}
Resumen: La Maestría en Educación Rural Centroamericana se propone ante la comunidad nacional e internaciEste artículo tiene como propósito reflexionar acerca de las realidades que viven las comunidades rurales de nuestro país, con miras en buscar soluciones a las problemáticas que éstas afrontan. Para ello, se hace un análisis desde diferentes perspectivas.

Ante este contexto, se toma en cuenta la importancia que tienen las comunidades rurales para el desarrollo de un país, el papel que juega la escuela como parte de éste, la forma en que las comunidades luchan por lograr una mejor calidad de vida y a través de la organización comunitaria.
\end{abstract}

Palabras clave: Comunidad rural, desarrollo rural costarricense, calidad de vida.

\begin{abstract}
The purpose of this article is to reflect about current reality experimented in rural communities in our country in order to look for solutions to their problems that allow them to reach a better quality of life. Analyses are made from different perspectives.

Within this context, we take into account the role that rural communities have in the development of a country as well as the part education plays in the fight for the achievement of a better quality of life.
\end{abstract}

Key words: Rural community, rural development, quality of life.

\section{INTRODUCCIÓN}

Los habitantes de zonas rurales son afectados por una serie de dificultadesque limitan su desarrollo integral. El aislamiento geográfico, las pocas fuentes de trabajo y la falta de aplicación de programas comunitarios acordes con las necesidades reales de estos pobladores, son algunos de ellos, lo que provoca grandes dificultades para obtener un mejoramiento de la calidad de vida, pues les es difícil obtener ingresos por lo que producen sus tierras; y el comercio no se interesa por pagarles los productos a un precio justo, aunque sean de buena calidad; es decir, se da la explotación de mano de obra, de tierras y de productos agrícolas. Los campesinos no tienen ninguna otra entrada

Educadora, graduada en la Universidad de Costa Rica (UCR) en Diplomado. Cursó sus estudios de Profesorado y Licenciatura en la Universidad Nacional (UNA). Máster en Educación Rural Centroamericana, Universidad Nacional (UNA). Ha trabajado como directora y

docente de una escuela ubicada en una comunidad rural. Correo electrónico: erboha75@yahoo.com 
y se tienen que conformar con lo que les paguen, lo que provoca que no se dé un claro desarrollo en las comunidades rurales. Por ello, es importante fortalecer la identidad rural de estas comunidades, para generar estrategias de desarrollo mediante la integración de los habitantes rurales, el conocimiento, el reforzamiento de valores y el desarrollo de destrezas relacionadas con la apreciación de recursos del medio y su utilización efectiva.

Según el análisis presentado por el (Instituto Interamericano de Cooperación para la Agricultura [IICA], 1999), en el medio rural se espera que el empleo agrícola y no agrícola contribuya, de forma más decidida, a la generación de ingresos para las comunidades rurales, gracias a la definición de políticas de desarrollo rural y a la creciente feminización de la agricultura.

Ante el contexto descrito, se valora el papel que podrían jugar las TICs en respuesta a las necesidades educativas del medio rural costarricense, y de esta forma, ampliar las posibilidades de desarrollo personal de estudiantes, de educadores y de las comunidades completas.

\section{FACTORES QUE AFECTAN A LOS HABITANTES DE ZONAS RURALES}

Las comunidades rurales son afectadas por factores que limitan su desarrollo y provocan que éstas vivan sometidas a las decisiones de los terratenientes o de los grandes empresarios. Algunos de estos factores son los siguientes:

- Vías de comunicación en mal estado, las cuales, muchas veces, son aún más dañadas por inundaciones o factores propios del clima. Además, estas vías carecen de mantenimiento adecuado.

- $\quad$ Calidad de servicios básicos: muchas comunidades carecen de agua potable y utilizan agua de fuentes propias de la finca o de acueductos rurales. Existen algunos pueblos que ni siquiera tienen acceso a ríos, por lo que construyen pozos en condiciones insalubres.

- $\quad$ Problemas con el transporte: funciona, casi siempre, de una manera irregular y en condiciones desfavorables para los habitantes de la comunidad, pues se encuentran serias dificultades para transportar los productos que comercian o para trasladarse hacia otros lugares a suplir sus necesidades.

De acuerdo con Ooijens (1997), es necesario promover el desarrollo sostenible, redefinir la tendencia de la tierra y estimular el uso más eficiente de otros recursos, tales como el crédito, la asistencia técnica y la comercialización, mediante la adaptación de los programas educativos a las necesidades locales. En estas zonas, básicamente, existen dos problemas recurrentes, a saber:

- Carencia de efectivos programas comunitarios por parte de los organismos locales, institucionales y gubernamentales: no ayudan a fomentar el desarrollo o no responden a las necesidades reales de las comunidades. Los que existen, casi siempre, son programas foráneos traídos por otras personas, la mayoría de las veces, incapaces de articular procesos sostenibles de desarrollo.

- $\quad$ Fuentes de empleo. En estos pueblos, las tierras se encuentran en manos de hacendados, los cuales contratan a una o dos familias para que le den el mantenimiento a la propiedad. Las demás son familias con fincas muy pequeñas, lo cual no les permiten generar empleo. Esta situación trae como consecuencia un estado de pobreza campesina, pues, por un lado, no 
pueden vender sus productos, y por otro, no encuentran un empleo con un salario digno para llenar sus necesidades y las de su familia.

Según el Noveno Informe del Estado de la Nación (Programa Estado de la Nación en Desarrollo Humano Sostenible, 2003), se documenta, de forma clara, la falta de asociación entre el crecimiento de los sectores productivos y su capacidad para generar empleos. Este texto dice, específicamente, que la insuficiencia en la generación de empleos de calidad, aunada al aumento en la desigualdad en el ingreso, son dos de las posibles razones por las que no ha disminuido la pobreza en Costa Rica.

Por lo anterior, es importante que en las zonas rurales se produzcan mayores tasas de crecimiento económico y se generen empleos según las fortalezas que sus comunidades tengan; esto les permitirá crear oportunidades para todas las zonas rurales y disminuir la pobreza. El Programa Estado de la Nación en Desarrollo Humano Sostenible (2004) argumenta que durante la década de los 90, las oportunidades de empleo de la población no mejoraron significativamente.

Según este documento, para el año 2000, de los 13,7 millones de ocupados en Centroamérica, un $39,3 \%$ lo estaba en el sector informal, un 30,6\% en actividades agropecuarias y un 30,1\% en el formal. En el sector agrícola, un 18,7\% del empleo corresponde a la actividad realizada por cuenta propia y familiares, que es un tradicional reducto de pobreza; un 5,4\% a pequeñas fincas que emplean un máximo de cinco trabajadores, y sólo un 6,6\% corresponde al sector agropecuario moderno, que son medianas o grandes fincas, con aplicación de tecnología y mecanización, y que emplean un número alto de obreros.

El mayor problema se presenta en los trabajadores campesinos por cuenta propia, entre quienes la pobreza alcanza el 74,3\%, así como entre patronos y asalariados agropecuarios, en fincas de cinco o menos empleados, con el 66,4\%. Además, cabe resaltar que según datos del Instituto Nacional de Estadística y Censos [INEC] (2001), la incidencia de la pobreza total se ha estancado entre un $18,5 \%$ y un $21,7 \%$ desde 1994 , y la pobreza extrema ha oscilado alrededor del $5 \%$, lo que significa que cada año miles de costarricenses ingresan en el grupo de personas pobres, si se tiene en cuenta el crecimiento anual de la población. Desde un punto de vista ético, la continuidad de esta situación resulta inaceptable y obliga a revisar, integralmente, los programas de inversión social y las políticas contra la pobreza.

La débil relación entre crecimiento y empleo, así como la falta de vinculaciones entre las nuevas actividades exportadoras y el resto de la economía, son los desafíos más importantes que deben encararse para asegurar un empleo de mejor calidad (más calificado y mejor pagado), así como la inclusión de las pequeñas y medianas microempresas en la dinámica económica. Es necesario hacer notar que los programas gubernamentales que se están desarrollando en las comunidades rurales, no llenan las expectativas o las necesidades reales de dichos pueblos.

Otro problema, es la pérdida de recurso humano, ya que los jóvenes se trasladan a comunidades urbanas a estudiar y trabajar, y la mayoría de ellos, no regresa a su lugar, sino que emigra hacia otras zonas.

Por otro lado, se debe mencionar que en casi todas las comunidades rurales se cuenta con servicio de un acueducto rural, un puesto de Equipos Básicos de Atención Integral en Salud (EBAIS), corriente eléctrica; un pobre servicio de transporte, una pulpería y un teléfono público.

En el campo laboral, la mayoría de las madres de familia trabajan en los quehaceres del hogar; otras, que son madres cabeza de familia, trabajan en las bananeras. En los casos en los que hay padres de familia, éstos también trabajan en las zonas bananeras como peones. 
Estas comunidades rurales reciben, algunas veces apoyo económico y de asesoría de algunas instituciones como el Instituto de Desarrollo Agrario (IDA), el Instituto Mixto de Ayuda Social (IMAS), el Instituto Nacional de Aprendizaje (INA) y otras, como la Mutual Cartago de Ahorro y Préstamo (MUCAP). Esta última les brinda la oportunidad de adquirir bonos de vivienda con apoyo a su forma de vida; pero aun así, por su situación económica, se les dificulta mejorar su calidad de vida.

Existen propuestas de desarrollo comunitario; ejemplo de ellas es el resumen que plantea la "Unión de Comunidades en Restauración Ecológica, Económica y Social”, en la que se enfatiza una Propuesta para el desarrollo de la agricultura familiar, con los siguientes componentes:

\section{Mejoramiento de la base productiva de nuestras fincas:}

- $\quad$ Mejoramiento de nuestras fincas para aumentar su competitividad, su productividad y su sostenibilidad.

- $\quad$ Actividades productivas para el mercado nacional, la exportación y los mercados de nichos.

- Acceso a la información y la innovación, para facilitar los cambios requeridos y enfrentar los desafíos actuales.

\section{Aumento del valor agregado}

- Valorización de nuestra capacidad para comercializar nuestros productos.

- Transformación de la producción en el ámbito de las fincas y las comunidades.

\section{Entorno de la producción}

- $\quad$ Protección del medio ambiente para promover la sostenibilidad.

- $\quad$ Turismo rural como actividad no-agrícola y herramienta para el desarrollo rural.

\section{Acompañamiento de nuestro desarrollo}

- Acceso al financiamiento con condiciones adaptadas a nuestras condiciones para desarrollar nuestras actividades.

- $\quad$ Educación de nuestros hijos.

- $\quad$ Fomento del desarrollo territorial rural para una prosperidad más equitativa.

- Nuevas políticas públicas y el apoyo institucional para fomentar la agricultura familiar.

\section{FORTALECIMIENTO DE NUESTRAS ORGANIZACIONES: ELEMENTO CLAVE DEL DESARROLLO}

Estos planteamientos tratan de fortalecer las comunidades rurales para que alcancen una mejor calidad de vida mediante la participación de todos los miembros de familia; con esto, se persigue que todos se involucren en el proyecto y sean beneficiarios de éste. 
Existen algunas instituciones que se interesan por el bienestar de las comunidades, entre ellas el IDA, con su programa de formación de asentamientos; el cual tiene como propósito adjudicarles tierras -en las categorías de parcelas, granjas y lotes- a los campesinos de zonas rurales que cumplan con los requisitos institucionales.

En Costa Rica, el Ministerio de Agricultura (Costa Rica. Ministerio de Agricultura, 2001) plantea algunos logros y vinculaciones de sus acciones con el desarrollo del medio rural, y se fundamenta que la participación de los diversos actores sociales, es de vital importancia para la capacidad de gestión, los niveles de organización y el desarrollo del conocimiento con que éstos cuentan.

Estos logros están orientados a fortalecer las capacidades de la población rural, a fin de contribuir con un mejor nivel de vida de las familias, por medio del fortalecimiento de los procesos organizativos, de la identificación de alternativas productivas con potencial, de la negociación de recursos diversos, de las acciones para mejorar el acceso a los servicios para el desarrollo y de la puesta en marcha de actividades productivas de bienes y servicios.

Debido a la amplitud del tema y a la complejidad de la realidad que vive la población del medio rural, se han establecido relaciones de coordinación con diversas instituciones públicas, más allá de las que componen el sector agropecuario, entre las que están: el Ministerio de Obras Públicas y Transportes (MOPT), el Instituto Costarricense de Turismo (ICT), el Instituto Nacional de Aprendizaje (INA), la Caja Costarricense de Seguro Social (CCSS) y el Ministerio de Educación Pública (MEP).

Se han impulsado diversas actividades de producción agropecuaria, tales como proyectos de viveros, granjas avícolas, cultivos diversos, especies menores, las que están a cargo de organizaciones de productores (hombres, mujeres, jóvenes, indígenas) con el apoyo de otras instituciones del sector agropecuario. Otra actividad que se impulsa es el turismo rural, en el que destaca la venta de servicios turísticos por Internet y el fortalecimiento de ésta en la región brunca, coordinada con las Cámaras de turismo y de algunas empresas agroindustriales; esto como resultado, especialmente, de los proyectos de desarrollo rural integral, tales como INPALSUR en la zona sur, algunas en la región huetar norte apoyadas por los Pequeños Productores de la Zona Norte (PPZN) y en el área del DRIP.

Existen otros proyectos no agropecuarios, tales como artesanías y ventas de comida, además de proyectos con un enfoque de conservación y uso racional de los recursos naturales, ubicados en cuencas hidrográficas. Por otra parte, conscientes de que el medio rural, para desarrollarse, requiere de servicios básicos, se impulsan programas de mantenimiento y rehabilitación de la infraestructura vial rural, por medio de convenios como el de la AMACARUVA, con el Ministerio de Agricultura y Ganadería (MAG), MOPT con participación de la Junta de Administración Portuaria y Desarrollo Económica de la Vertiente Atlántica (JAPDEVA), para el mantenimiento y la rehabilitación de caminos rurales en esta zona.

Además, se proponen acciones para el mejoramiento de la infraestructura comunal, por medio de la gestión de recursos para la construcción de escuelas y centros de salud, el fortalecimiento de la capacidad de gestión de las municipalidades, el apoyo y la asesoría a procesos de formulación y negociación de proyectos de acueductos rurales, rellenos sanitarios y manejo de deshechos sólidos, programas de capacitación y asesoría tendientes a fortalecer la capacidad gerencial, y acciones y convenios para incidir en la educación que se desarrolla en el medio rural, mediante proyectos como el de instalación del Sistema de Información del Sector Agropecuario Costarricense (InfoAgro) en colegios técnicos con modalidad agropecuaria, y la conformación de centros de información y gestión empresarial, en coordinación con el MEP, el IICA, el Consejo Nacional de Producción (CNP) y el Triángulo de Solidaridad. 
Todos estos servicios han contribuido de forma muy positiva con las comunidades rurales del país, en campos tan importantes como la salud, esto mediante campañas en comunidades específicas, especialmente algunas indígenas, en coordinación con la CCSS y clínicas privadas.

Otros aportes tienen que ver con gestión de información dentro de los grupos e instituciones, por medio de eventos de acercamiento entre la oferta y la demanda de recursos técnicos, financieros y de capacitación, en el nivel local y nacional, así como con actividades para fomentar y facilitar los procesos de comercialización de productos, por medio de ferias, como EXPOPOCOCI, la cual se realiza anualmente, y otras iniciativas como convenios con instituciones y organizaciones que tienen experiencia en esta temática. Éstas y otras actividades son de gran impacto, pues han permitido que el Programa de desarrollo rural haga su aporte al desarrollo rural del país.

Además de lo anterior, se plantean microproyectos educativos, mediante los cuales se motiva a los pobladores para que, de acuerdo con sus necesidades, se organicen y hagan producir sus tierras, utilicen sus recursos y sus habilidades para obtener beneficios socioeconómicos que les permitan adicionar ingresos a sus familias.

También se motiva a los grupos de mujeres jefas de hogar, productoras organizadas y jóvenes, las que se orientan a la organización y la planificación de proyectos agroproductivos; los cuales ayudan a mejorar la organización comunitaria en los campos económico, social, político y ambiental, para lograr una mejor calidad de vida, mantener su identidad cultural y, sobre todo, encontrar fuentes de trabajo en su propia comunidad.

Las iniciativas para el desarrollo del medio rural se fundamentan en la participación de los diversos actores sociales, pero son de vital importancia la capacidad de gestión, los niveles de organización y el conocimiento con que éstos cuentan; por medio del fortalecimiento de los procesos organizativos, la identificación de alternativas productivas con potencial, la negociación de recursos diversos, las acciones para mejorar el acceso a los servicios para el desarrollo y la puesta en marcha de actividades productivas de bienes y servicios.

Para alcanzar todo lo proyectado, debemos ser conscientes de que el medio rural requiere de servicios básicos, como por ejemplo: el impulso de programas de mantenimiento y de rehabilitación de la infraestructura vial rural, por medio de convenios para bien comunal con instituciones como el MAG, el MOPT y la participación de JAPDEVA.

Es pertinente señalar el significativo papel que tiene el sector agropecuario en el desarrollo rural que, aunque aún necesita mejorar; sólo podrá hacerlo lograr en la medida en que se le permita crear nuevas oportunidades en un contexto de crecimiento económico, equitativo, competitivo y sostenible. Se pretende buscar procesos que se dirijan hacia la construcción de capacidades en las instituciones y organizaciones de las comunidades, con el propósito de reducir la vulnerabilidad de las poblaciones del medio rural.

Para ello, serán importantes las capacitaciones mediante conferencias, revisión de temas y textos de las charlas, convocatorias y cursos de aprovechamiento teóricos y prácticos. Además, se debe fomentar entre los habitantes de zonas rurales, la toma de conciencia acerca de que ellos son capaces de ser líderes, de adquirir nuevos conocimientos y de adoptar actitudes hacia una mentalidad más empresarial y socioeconómica, manteniendo las estructuras económicas y los valores culturales, y proporcionando la inserción en los mercados internos y externos con ayuda de recursos propios.

Es importante conservar el uso de los recursos naturales y humanos de los sistemas de producción y elevar el nivel de gestión empresarial de las organizaciones de productores campesinos, según sus necesidades; de manera equitativa, sostenible y competitiva, preservando los recursos 
naturales y fortaleciendo la participación de los niños, las niñas y los padres en general. Asimismo, se debe contribuir a fortalecer y facilitar el conocimiento, las técnicas y la metodología, mediante un modelo participativo comunitario.

Es necesario impulsar acciones generadoras de propuestas y articular procesos para que el desarrollo rural mantenga equilibrio en los ámbitos social, económico y ambiental, por medio de una estrategia que permita la participación de cada uno de los miembros de las comunidades rurales. Se debe conservar una organización, una gestión y una negociación que impulse el bienestar de todos, en condiciones de equidad.

Las anteriores son algunas razones por las cuales se quiere integrar la escuela con la comunidad en microproyectos, los cuales permitan solventar necesidades, así como aprovechar más sus recursos naturales $\mathrm{y}$, de esta forma, obtener ganancias.

Algunas instituciones han luchado por el mejoramiento de estas comunidades rurales, y tratan de solventar su situación socioeconómica, como en el caso de el Plan Nacional de Desarrollo en Marcha (Costa Rica. Ministerio de Planificación y Política Económica [MIDEPLAN] 1994-1995, 2001), que busca promover cambios de actitud que impliquen modificaciones en las formas de vida de los campesinos rurales, buscando mejorar los patrones de consumo y los esquemas productivos, con el propósito de elevar el grado de conciencia sobre los problemas ambientales y promover una genuina actitud de responsabilidad entre estos.

Las acciones desarrolladas con este propósito se orientan a fortalecer y a promover el desarrollo sostenible, y a ratificar e implementar los acuerdos internacionales relacionados con la promoción del desarrollo sostenible de las comunidades rurales.

Los siguientes son algunos proyectos comunitarios.

- Uso de tecnologías limpias.

- $\quad$ Sistemas de certificación ambiental.

- $\quad$ Agricultura orgánica.

- Apoyo a iniciativas privadas para el manejo de desechos, recolección, clasificación y reciclaje.

- $\quad$ Manejo de desechos sólidos.

- $\quad$ Promoción y cambio de actitud.

La Escuela Agrícola Regional del Trópico Húmedo (EARTH) también ha implementado programas de desarrollo comunitario desde 1995. Desarrollar trabajo con las comunidades ha permitido que este programa tenga una visión cada vez más profunda, de las necesidades de los grupos, de las organizaciones y de las personas de la región Atlántica. Esta Institución ayuda a las comunidades rurales con proyectos de capacitación dirigidos a grupos u organizaciones comunales, familias rurales y escuelas, los que definen los temas de su capacitación de acuerdo con sus intereses y necesidades.

El INA también ha dado su aporte en las comunidades rurales con proyectos que han generado grandes desafíos en la agricultura, para motivar a las comunidades que han perdido el interés sobre sus fincas. Para ello, las instituciones y las personas involucradas se han propuesto una tarea colectiva de unir esfuerzos e influir sobre el futuro de las comunidades rurales.

Por otra parte, se debe destacar que las familias campesinas están enfrentado el proceso de globalización, por lo que sus condiciones de vida son cada vez más difíciles. Les preocupa el futuro de sus hijas e hijos, y, sobre todo, el futuro de la agricultura, ya que la mayoría de ellas y ellos no quieren seguir en esta actividad y, muchas veces, ni siquiera desean seguir viviendo en la comunidad. 
El Programa Estado de la Nación (2000) hace notar la situación que enfrentan las comunidades rurales. Los ocupados por cuenta propia y en microempresas en el ámbito regional, superan, ligeramente, la ocupación formal (31,1\% y 30,1\%, respectivamente), lo que evidencia la importancia de estas labores. Por su parte, las actividades agropecuarias corresponden, en su mayoría, a la agricultura tradicional realizada por personas que trabajan por cuenta propia (no tienen patrón) y trabajadores familiares (18,7\%), más los asalariados y patronos en explotaciones agrícolas de cinco empleados o menos $(5,4 \%)$. En contraste, sólo un 6,6\% de los centroamericanos ocupados realiza actividades en el sector agropecuario moderno es decir, en fincas o explotaciones agrícolas con más de cinco trabajadores, mecanizadas y con aplicación de tecnología.

Respecto a la situación por género, un 36,2\% del total de ocupados en la región son mujeres, lo que equivale a cinco millones de centroamericanas que participan en forma activa en el mercado laboral. Trabajan, especialmente, en el sector informal, pues casi tres de cada cinco ocupadas se desempeñan ahí (59,3\%). Asimismo, el agropecuario es importante, sobre todo, para los hombres, de los cuales un 42,4\% está ocupado en el sector.

El sector formal tiene la misma importancia relativa para ambos sexos, ya que ocupa alrededor del $30 \%$ en cada caso. Sin embargo, dada la diferencia en el número absoluto de hombres y mujeres que participan en el mercado de trabajo, hay dos aspectos que deben resaltarse. Por una parte, el número de mujeres en el sector informal supera al de los hombres (2,9 millones de mujeres respecto a 2,4 millones de hombres). Por otra, aunque el sector formal es igualmente importante, en términos relativos, para hombres y mujeres, hay 1,7 hombres ocupados por cada mujer.

Ahora bien, los ingresos que obtienen los ocupados en los sectores informal y agropecuario tradicional, generalmente son bajos, lo que ocasiona una situación de pobreza en estas personas y sus familias. La mayor incidencia de la pobreza se presenta entre los que trabajan en el sector agropecuario: un 69\% de ellos, que representan un 49,6\% de todos los trabajadores pobres. Se pone en evidencia, entonces, que el alivio de la pobreza requiere, necesariamente, mejorar la situación de los campesinos y sus familias. Son muchos los problemas que enfrentan los campesinos, pero sobresale la explotación que se da en el trabajo y en la producción agrícola, lo que ha incrementado el empobrecimiento en estas familias.

En nuestro país, existen instituciones gubernamentales y no gubernamentales que se preocupan por mejorar calidad de vida de nuestras comunidades rurales, desde la generación de competencias que contribuyan a la autogestión de proyectos productivos, mediante procesos participativos que involucren aspectos culturales de su contexto.

\section{LA ESCUELA RURAL Y LOS MICROPROYECTOS SOCIOECONÓMICOS}

Es importante recalcar que existen también otras maneras que permiten al habitante de zonas rurales solventar algunas de sus necesidades, por ejemplo: la escuela rural que es parte fundamental de una comunidad en proceso de desarrollo, ya que en ella, mediante la implementación de microproyectos socioeconómicos, se adquieren estrategias para lograr un mejor funcionamiento comunitario, para desarrollarse y aplicar sus habilidades y actitudes. Es importante que los miembros de la comunidad se conozcan mejor, saber cuáles son sus intereses y sus necesidades, cuál es el arraigo que tienen por su identidad cultural y, sobre todo, cuál es su interés por defenderlas.

La escuela rural juega un papel importante en esta investigación, pues relaciona a los habitantes de la comunidad con los proyectos planteados, en contraposición de una serie de factores 
que han frenado el desarrollo comunal, entre ellos: la desintegración familiar, la incorporación de la mujer al proceso laboral y la incorporación de los niños al trabajo desde temprana edad.

Según Ooijens (1998) citado por Aguilar (2000), éstos son algunos factores que limitan la función socializadora de la familia y de la escuela, y constituyen, también, razones para promover cambios significativos en la educación, la escuela, la comunidad y la familia, los cuales se orientan a fortalecer la participación y la solidaridad.

Recordemos que la escuela está formada por todos los miembros de la comunidad, sin importar el vínculo que los una. Debe darse una real participación de cada uno de ellos, de forma que les permita mejorar su condición de vida y se establezca una estrecha relación entre la escuela y toda la localidad.

Cabe destacar que las comunidades rurales son afectadas por una serie de desventajas, como por ejemplo, el desestímulo a la producción en lo que refiere a granos básicos; la dificultad de acceder al crédito y al seguro de cosechas, los altos costos de producción y la falta de servicios estratégicos.

Aguilar (2000), considera que, a pesar de que en las zonas rurales se han diversificado las fuentes de empleo y se han creado espacios para impulsar nuevas actividades y relaciones con diferentes mercados, se mantiene la pobreza, pues faltan condiciones adecuadas para la producción y existen problemas de infraestructura y de servicios; asimismo, se mantienen bajos índices en salud y en educación, y se ha propiciado la extinción del pequeño propietario.

\section{COMUNIDADES RURALES}

Según Aguilar (2000), las comunidades rurales están compuestas por un grupo de niñas y niños, jóvenes, adultos mayores, hombres y mujeres en su dimensión social y organización familiar, con formas de vida campesina y una identidad cultural particular.

La integración entre ellos debe funcionar constantemente, ya que la prosperidad de un lugar depende de la relación que se establezca entre las personas. Debe existir un trabajo en conjunto para que se logren las metas propuestas.

\section{Calidad de vida}

Un elemento intrínseco de la estrategia aplicada a la calidad de vida consiste en cultivar ese sentido de pertenencia y de cohesión social a una comunidad; la estrategia tiene por eje la participación activa del conjunto de la comunidad en su desarrollo y en la formación permanente, y la educación, escolar y extraescolar de todos los miembros.

En sus actividades a favor de las poblaciones con bajos ingresos, la Fundación Noor alHussein (NHH), importante organización no gubernamental de Jordania, adoptó el principio de un desarrollo socioeconómico global, con un enfoque interdisciplinario. El proyecto "Calidad de vida" contempla lo que necesita cada comunidad para su desarrollo, en particular, en materia de salud, nutrición, ambiente y educación. Este proyecto se materializa en programas de perfeccionamiento de los recursos humanos, los cuales aportan a las comunidades los conocimientos, la educación y las competencias que necesitan, en asociación con los padres y los dirigentes de una comunidad como interlocutores de los mecanismos establecidos. 


\section{Habilidades y actitudes}

En el ámbito social, se desarrollan nuevas actitudes y aptitudes frente a lo tecnológico, el mercado, la información y las inversiones.

Román (1998), mencionada por Aguilar (2000), señala que se ha dado un cambio en los hábitos de vida, sean éstos de consumo, de períodos de trabajo, de plazos de entrega de los productos, de períodos de descanso, de prácticas de cultivo, de significado de las prácticas y de los valores competencia, solidaridad y riesgo, de seguridad en las aspiraciones y de las expectativas de desarrollo.

Para que el habitante rural crezca, debe desarrollarse y potenciar sus capacidades, aprender, generar conocimientos, desarrollar destrezas, habilidades y valores que le posibiliten un mejoramiento real de sus condiciones de vida.

\section{Organización comunitaria}

Según el Proyecto de Comayagua, Honduras, "Educación para el Trabajo" (POCET, 1991), a pesar de que es poca e incipiente la organización de los pequeños productores, se forman organizaciones con un carácter más regional, las cuales, algunas veces, se reúnen en federaciones nacionales. Esta organización formal no solamente aspira a combinaciones económicas más eficientes, sino también a establecer sistemas socialmente más equitativos y autónomos.

La organización de los pequeños productores agrícolas de las comunidades rurales es elemental para alcanzar diversos objetivos en su desarrollo. Cuando una comunidad rural se organiza, obtiene grandes logros, como por ejemplo, recursos financieros, capacitación y un mejor aprovechamiento de los recursos de su entorno, disposición de mejores servicios económicos, educación y vivienda y otros.

En el informe técnico del proyecto de educación para el trabajo se plantea que la organización comunal es un conjunto de empresas y de organizaciones formadas por trabajadores que sostienen la primacía del trabajo. Optan por la propiedad social de los medios de producción y establecen que el excedente generado es un medio para elevar el nivel de vida de sus miembros.

\section{Microproyectos}

Los microproyectos son pequeñas iniciativas que nacen de la realidad comunal y se concretan a partir de un diagnóstico. Permiten la realización de vivencias que conjugan la práctica del conocimiento, las habilidades, las actitudes y los valores necesarios para la convivencia humana.

Son un medio para la integración comunal, pues surgen de una situación económica real, ya sea un problema, una fortaleza, una debilidad o una necesidad de una comunidad específica. Estos permiten el desarrollo de múltiples conocimientos que producen grandes ingresos socioeconómicos relacionados con la situación que da origen a cada microproyecto ideado.

\section{Identidad cultural}

La pérdida de identidad cultural, como consecuencia de la orientación de la sociedad hacia patrones de desarrollo urbano-industrial, está cambiando el papel de lo rural dentro del panorama de 
relaciones entre rurales y urbanos: pierde, significativamente, su función como productor de alimentos y se convierte en un entorno de reducida calidad de vida por insuficiencia en la dotación de servicios esenciales y bajos niveles de renta; y ha pasado a ser un lugar de atracción residencial y de demanda de componentes ambientales de esparcimiento, descanso y ocio. Esta circunstancia aparece más como una oportunidad que como un problema, pero siempre y cuando ese «retorno» al medio rural se realice sin una invasión destructiva de los valores y los recursos más preciados por quienes la visitan.

En opinión de van der Bijl (2000), la gente adopta, cada vez más, valores urbanos, entre los que están el individualismo, el consumismo y la competencia, y pierde su identidad cultural.

En las comunidades rurales se pierde gran cantidad de recurso humano, pues aunque logran estudiar en el colegio o en la universidad, no consiguen insertarse laboralmente en su comunidad, y optan por emigrar a las ciudades. Esta decisión contribuye a que se pierda la identidad cultural, ya que las familias se adaptan a otra forma de vida.

van der Bijl (2000) argumenta que el desarrollo de una zona rural debe nacer de la misma población, y que debe ser, ante todo, un desarrollo desde la comunidad. Este autor señala la necesidad de que la escuela les brinde a los miembros de estas zonas, capacitación y técnicas para la iniciativa y la organización. Considera que el principal desafío de la escuela es convertirse en una opción interesante y atractiva para los grupos mayoritarios en el campo, con una educación adecuada a su realidad y a sus necesidades, y que ofrezca instrumentos que les ayuden a mejorar su situación económica y social.

\section{Organización comunal}

La mayoría de los habitantes de zonas rurales ve limitadas sus posibilidades por la falta de organización comunal.

Hay que mencionar que en las áreas rurales de Costa Rica, los niveles de organización son muy bajos, por lo que pocas veces sobresalen acciones autogestionarias; a esto hay que agregar el hecho de que el liderazgo suele quedar en manos de personas provenientes del exterior de la comunidad, como el pastor, el sacerdote y el maestro (Aguilar y Monge, 2000).

Es interesante saber que en estas zonas se concentra la mayor cantidad de riqueza natural y de materia prima. Pero hay que cuestionarse sobre los motivos que generan la falta de organización y las consecuencias negativas que este hecho produce en el proceso de su desarrollo socioeconómico.

\section{Desarrollo comunitario}

Es un proceso de formación y de consolidación de organizaciones de productores. Tiene como objetivo analizar los procesos que dieron origen a las diversas formas de vida de las comunidades rurales. Con el desarrollo comunitario se establecen las estrategias de formación, la evolución de las organizaciones, la creación y fortalecimiento de las organizaciones, la proyección de proyectos para potenciar el bienestar de la comunidad y favorecer a cada uno de los pobladores rurales.

Según Gaviria (2002), desarrollo comunal es la organización empresarial autogestionaria conformada por los miembros de una comunidad que se agrupan para producir bienes o servicios. 
Las necesidades educativas de las zonas rurales y el potencial subutilizado, en términos de experiencias vividas, de producción documental y de recursos disponibles por el otro, han llevado a la necesidad de construir un espacio que les permita a los actores identificados, unir sus esfuerzos en busca del desarrollo de las comunidades rurales. Cabe destacar que estrechar relaciones entre los miembros de estas comunidades es importante, pues eso les ayuda a organizarse, a perder el temor de luchar por sus ideales y a fortalecer el trabajo en equipo.

Es necesario tener presente que debe existir el diálogo y el intercambio de conocimientos para que todos se sientan útiles, mejoren su autoestima y vean lo que pueden lograr con una organización comunal que fomente el arraigo cultural y la transmisión de costumbres, valores y tradiciones del pueblo. Al generar actitudes de identidad rural y aprecio por los recursos propios, utilizarlos adecuadamente, tomar conciencia ecológica, desarrollar un mayor apego a la diversidad natural de estas zonas, y generar actitudes que promuevan el desarrollo sociocultural, favorece a toda la comunidad.

El reto principal que debemos asumir los habitantes de una zona rural es influir, positivamente, en la organización comunitaria, para un desarrollo en los educandos en sus comunidades. Cuanta más humana sea la organización, mejores y mayores serán los logros que se alcancen en éstas.

\section{REFERENCIAS}

Aguilar, M. E. y Monge, M. (2000). Hacia una pedagogía rural. Heredia: EUNA.

Costa Rica: Ministerio de Agricultura y Ganadería [MAG]. (2001). Memoria 2000. Presentada a la Asamblea Legislativa por el Ministro Ing. Alberto Dent Zeledón. San José: Autor.

Costa Rica. Ministerio de Planificación Nacional y Política Económica [MIDEPLAN].(2001). Plan nacional de desarrollo humano. La ruta hacia un nuevo modelo de desarrollo. San José: Autor.

Gaviria, A. (2002). Grupos organizados. OIMT. Actualidad forestal tropical. 10/4, 14-15. Consultado el día, mes, año de http://www.itto.or.jp/live/Live_Server/187/tfu.2002.04(14-15).s.pdf

Instituto Interamericano de Cooperación para la Agricultura [IICA]. Dirección de desarrollo rural sostenible. (1999). Desarrollo rural sostenible en el marco de una nueva lectura de la ruralidad. Nueva ruralidad. San José, Costa Rica: Autor.

Instituto Nacional de Estadística y Censos (2001). "IX Censo Nacional de Población y V de Vivienda: Resultados generales". Consultado el 22, junio de 2006 de en http://www.inec.go.cr/ INEC_DIS/Publicaciones/archivos\%20SerieCensa1\%20xls/ResulCenso2000.pdf

Ooijens, J. (1997). La educación para la paz, la democracia y los derechos humanos. Elemento básico para la cultura de la paz en Centroamérica. Conferencia. San José, Costa Rica: UNESCO.

Programa Estado de la Nación en Desarrollo Humano Sostenible. (2000). Sexto Informe del Estado de la Nación en Desarrollo humano sostenible. San José: Programa del Estado de la Nación.

Programa Estado de la Nación en Desarrollo Humano Sostenible. (2003). Noveno Informe del 
Estado de la Nación. San José, Costa Rica: Programa Estado de la Nación.Programa Estado de la Nación en Desarrollo Humano Sostenble. (2004). Informe sobre desarrollo humano en Centroamérica y Panamá. Conozcamos más de Centroamérica. Desafíos económicos y de la integración. San José, Costa Rica: Autor.

Proyecto de Educación para el Trabajo. (1991). Comayagua, Honduras: POCET.

Román, I. (1997). Tendencias de cambio social en el proceso de modernización agrícola costarricense (1980-1996). Revista Perspectivas Rurales 1. Programa regional de Maestría en Desarrollo Rural, Heredia, Costa Rica: Universidad Nacional.

van der Bijl, B. (2000). Adecuación al contexto rural. División de Educación Rural, Universidad Nacional. 